\title{
FINITE ELEMENT MODELING OF THE NEURON-ELECTRODE INTERFACE: STIMULUS TRANSFER AND GEOMETRY
}

\author{
J.R. Buitenweg, W.L.C. Rutten and E. Marani \\ Institute of Biomedical Technology, Faculty of Electrical Engineering, University of Twente, \\ P.O. Box 217, 7500 AE Enschede, The Netherlands, email: J.R.Buitenweg@el.utwente.nl
}

\begin{abstract}
The relation between stimulus transfer and the geometry of the neuron-electrode interface can not be determined properly using electrical equivalent circuits, since current that flows from the sealing gap through the neuronal membrane is difficult to model in these circuits. Therefore, finite element modeling is proposed as a tool for linking the electrical properties of the neuronelectrode interface to its geometry.
\end{abstract}

\section{INTRODUCTION}

The electrical contact between a planar microelectrode and a cultured neuron depends on the geometry of the neuronelectrode interface and is of importance for effective stimulus transfer. In literature, electrical equivalent circuits are used frequently for modeling the electrical behaviour of the neuron-electrode interface [1,2]. Are these circuits suitable for studying the relation between stimulus transfer and the geometry of the neuron-electrode interface or do we need a more detailed finite element model?

\section{METHODS}

The geometry of the neuron-electrode interface is both mapped to the components of an electrical equivalent circuit and used for finite element modeling of the neuron-electrode

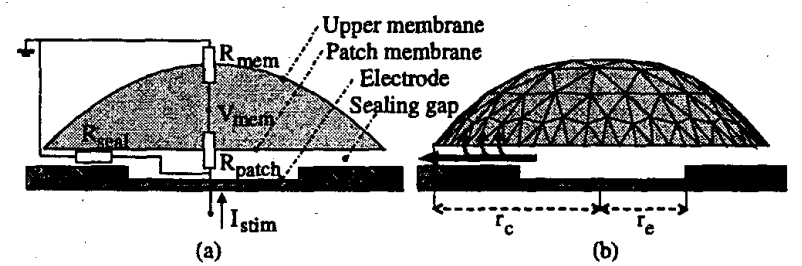

Fig. 1 Geometry of the neuron-electrode interface. (a) Electrical equivalent circuit. (b) Finite element model, implemented in ANSYS Finite Element Software (SAS IP inc, Houston) release 5.4.

contact (fig. 1). A stimulus current of $1 \mathrm{nA}$ is applied to the electrode. In the simulations, the radius of the neuron is varied from $r_{c}=7$ to $r_{c}=20 \mu \mathrm{m}$, and the radius of the electrode from $r_{e}=3 \mu \mathrm{m}$ to $r_{e}=5 \mu \mathrm{m}$. The computed stimulus transfer is defined as the maximum depolarisation of the upper membrane, due to the stimulus current.

\section{RESULTS}

In fig. 2, the simulation results from the finite element model are plotted together with the results from the circuit model. The results of the circuit model suggest that an optimal stimulus transfer exists for a certain radius of the neuron and

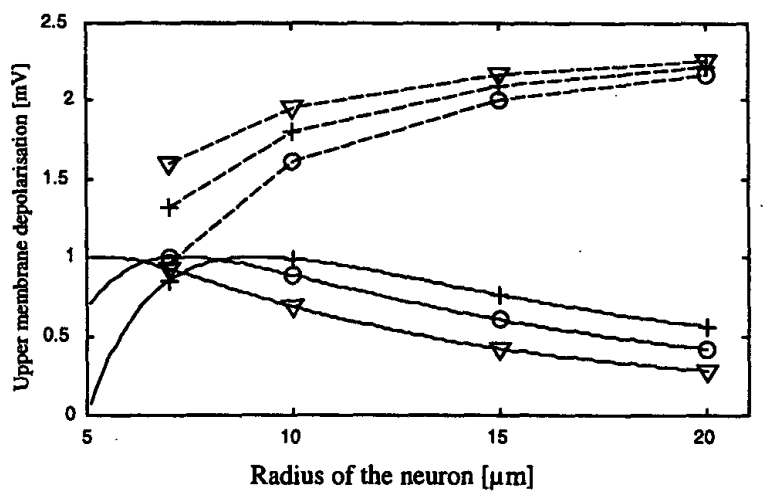

Fig. 2 Stimulus transfer as computed with the finite element model (dashed lines) and the circuit model (solid lines) plotted versus the radius of the neuron, $r_{c}$, for an electrode radius of $r_{c}=3 \mu \mathrm{m}$ (' $\nabla$ '), $r_{c}=4$ $\mu \mathrm{m}$ ('t') and $\mathrm{r}_{e}=5 \mu \mathrm{m}$ ('o').

that this optimum depends on the size of the electrode. The results from the finite element model, however, do not show this optimum.

\section{DISCUSSION}

The differences between the finite element model and the circuit model are due to the stimulus current that flows from the sealing gap through the lower membrane of the neuron (fig. 1b), which is implicitly modeled by the finite element model. Hence, the effective area of the patch membrane is larger than modeled in the circuit model, resulting in a larger stimulus transfer.

\section{CONCLUSIONS}

Finite element modeling is necessary for linking the electrical properties of the neuron-electrode interface to its geometry, since it accounts inherently for current flowing from the sealing gap through the neuronal membrane.

\section{ACKNOWLEDGEMENT}

This work was supported by the BIOMED II EC project, shared cost contract no. BMH4-2723

\section{REFERENCES}

[1] W.G. Regehr, J. Pine, C.S. Cohan, M.D. Mischke and D.W. Tank, J. Neurosc, Meth., vol. 30., pp. 91-106, 1989.

[2] J.R. Buitenweg, W.L.C. Rutten, W.P.A.Willems and J.W. van Nieuwkasteele, Med. Biol. Eng. Comp., vol. 36, pp. 630-637, 1998. 